\title{
LOCAL JORDAN ALGEBRAS
}

\author{
BY
}

\author{
MARVIN E. CAMBURN(1)
}

\begin{abstract}
A local Jordan algebra $\boldsymbol{S}$ is a unital quadratic Jordan algebra in which $\operatorname{Rad} 3$ is a maximal ideal, $3 / \operatorname{Rad} 3$ satisfies the DCC, and $\cap_{k} \operatorname{Rad} 3^{(k)}$ $=0$ where $K^{(n+1)}=U_{K}(n) K^{(n)}$. We show that the completion of a local Jordan algebra is also local Jordan, and if $\boldsymbol{S}$ is a complete local Jordan algebra over a field of characteristic not 2 , then either (1) 3 is a complete completely primary Jordan algebra, (2) $3 \cong 3_{1} \oplus 3_{2} \oplus S$ where each $3_{i}$ is a completely primary local Jordan algebra, or (3) $\Im \cong \$\left(D_{n}, J_{a}\right)$ where $(D, j)$ is either a not associative alternative algebra with involution or a complete semilocal associative algebra with involution.
\end{abstract}

The concept of local ring was first introduced for commutative associative rings which are Noetherian [2], and was later extended to noncommutative associative rings which are right Noetherian [1]. In this paper, we consider a class of quadratic Jordan algebras which in the linear associative case reduces to a class of local associative algebras which are not necessarily Noetherian [10]. In $\S 1$, some basic topological concepts are introduced, and in $\$ 2$, we form the completion of a local Jordan algebra showing that it is also a local Jordan algebra. In §3, we develop an idempotent lifting property of complete local Jordan algebras in order to show in $\S 4$ that the structure of these algebras in general is that of a Jordan matrix algebra over a complete semilocal associative algebra with involution.

1. Preliminaries. Let $M$ be a unital $\Phi$-module where $\Phi$ is a commutative associative unital ring. If there is a nonincreasing sequence of submodules $M=$ $M_{0} \supseteq M_{1} \supseteq M_{2} \supseteq \cdots$, then $\left\{x+M_{i}: x \in M\right\}$ is a basis for a topology in which addition and scalar multiplication are uniformly continuous. Also for any $S \subseteq M$, Cl $S=\bigcap_{0<i<\infty}\left(S+M_{i}\right)$ so that $S$ is closed $\Leftrightarrow S=\bigcap_{i}\left(S+M_{i}\right)$, and consequently, every open subset of $M$ is also closed. This topology is Hausdorff $\Leftrightarrow \bigcap_{0<i<\infty} M_{i}=0$. In case the induced topology is Hausdorff, a norm can be defined on $M$ by: $\|0\|=0$; and $\|x\|=e^{-k}$ if $x \neq 0$, where $x \in M_{k}-M_{k+1}$.

Presented to the Society, October 30, 1971; received by the editors January 6, 1972. AMS (MOS) subject classifications (1970). Primary 17C99; Secondary 16A10. algebra.

Key words and phrases. Quadratic Jordan algebra, radical, completion, Jordan matrix

(1) The results in this paper may be found in the author's dissertation completed under Marvin L. Tomber at Michigan State University. 
Then it is clear that $\|x+y\| \leqslant \max \{\|x\|,\|y\|\}$ and $\|\alpha x\| \leqslant\|x\|$ for $\alpha \in \Phi$. Finally, if $T$ is the topology induced by the sequence $\left\{M_{i}\right\}$, then $T$ coincides with the metric space topology derived from $d$, where $d(x, y)=\|x-y\|$.

Let $M^{*}$ be the unital $\Phi$-module which is the completion of $M$ as a metric space. Then $M^{*}$ has norm \|\|$^{*}$ where \|\|$^{*}$ restricted to $M$ yields \|\| . We note that the operations in $M^{*}$ are extensions of the uniformly continuous operations in $M$; that $N$ a submodule of $M \Rightarrow N^{*}=\mathrm{Cl}_{M^{*}}(N)$ is a submodule of $M^{*}$; and that every open submodule $P$ of $M^{*}$ is of the form $N^{*}$ where $N=N^{*} \cap M$ is an open submodule of $M$. Also the topology in $M^{*}$ is induced by the nonincreasing sequence of submodules $M_{0}^{*} \supseteq M_{1}^{*} \supseteq \cdots$ [12].

2. Local Jordan algebras. If $M$ is a unital $\Phi$-module with "product" $U$, where $U_{x} y$ is quadratic in $x$ and linear in $y$ and $1 \in M$ is a distinguished element such that $U_{1}=\mathrm{id}_{M}$, then $\Im=(M, U, 1)$ is a (unital) quadratic Jordan algebra providing the standard conditions are satisfied by $U$ and its associated bilinear mapping [4], [6]. When no ambiguity can arise, a quadratic Jordan algebra will be denoted simply by $\mathcal{Y}$. If $1 / 2 \in \Phi, \mathcal{Y}$ is a linear Jordan algebra under the bilinear composition $x \cdot y=1 / 2(x \circ y)=1 / 2 U_{x, y}$ [4].

An element $x \in \mathcal{Y}$ is invertible if there exists an element $y \in \mathfrak{Y}$ such that $U_{x} y=x$ and $U_{x} y^{2}=1$, and we write $y=x^{-1}$. If $z$ is an element of either an alternative algebra or a quadratic Jordan algebra, we say $z$ is quasi-invertible if $1-z$ is invertible. An ideal is quasi-invertible if every element is quasi-invertible. Throughout this paper the maximal quasi-invertible ideal of an appropriate algebra $\mathfrak{U}$ will be denoted by $\operatorname{Rad} \mathfrak{A}$.

We have the following facts concerning maximal ideals in a quadratic Jordan algebra 3 .

$\operatorname{Rad} \Im$ a maximal ideal $\Rightarrow \Im$ has exactly one maximal ideal.

$\Im / \operatorname{Rad} \Im$ is a Jordan division algebra

$\Leftrightarrow \operatorname{Rad} \mathcal{Y}=\{z \in \mathcal{Y}: z$ is noninvertible $\}$.

Note that (2.1) and (2.2) follow as in the associative case with the exception of $\Rightarrow$ of (2.2) which follows since $x$ invertible modulo $K, K$ a quasi-invertible ideal, $\Rightarrow U_{x} y=1-z, z \in \operatorname{Rad} \Im, \Rightarrow U_{x} y$ is invertible $\Rightarrow U_{x} \Im$ contains an invertible element $\Rightarrow x$ is invertible.

Let $\left\{K_{i}\right\}$ be a nonincreasing sequence of ideals of $\mathcal{S}=K_{0}$ with $\bigcap_{0<i<\infty} K_{i}=0$. Then, in addition to the two uniformly continuous module operations, $U$ is uniformly continuous. This follows since $x$ or $y$ in $K_{i} \Rightarrow$ $U_{x} y$ in $K_{i}$ and $x, y$ or $z$ in $K_{i} \Rightarrow U_{x, y} z$ in $K_{i}$ so 


$$
\left\|U_{x} y\right\| \leqslant \min \{\|x\|,\|y\|\} \text { and }\left\|U_{x, y} z\right\| \leqslant \min \{\|x\|,\|y\|,\|z\|\} \text {. }
$$

Thus

$$
\begin{aligned}
\left\|U_{x} y-U_{w} z\right\| & =\left\|U_{x}(y-z)+\left(U_{x}-U_{w}\right) z\right\| \\
& =\left\|U_{x}(y-z)+U_{x-w} z+U_{x-w, w} z\right\| \\
& \leqslant \max \left\{\left\|U_{x}(y-z)\right\|,\left\|U_{x-w} z\right\|,\left\|U_{x-w, w} z\right\|\right\} \\
& \leqslant \max \{\|x-w\|,\|y-z\|\} .
\end{aligned}
$$

The uniformly continuous operations in $\Im^{*}$ extend to operations in $\Im^{*}$,

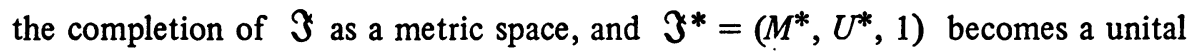
quadratic Jordan algebra.

The following lemma now follows easily.

LEMMA 1. Let $\Im$ have completion $3^{*}$ with respect to a sequence of ideals $\left\{K_{i}\right\}$. (1) If $A$ is an ideal of $\Im$, then $A^{*}$ is an ideal of $\}^{*}$. (2) The mapping $A=A^{*} \cap \Im \mapsto A^{*}$ is a bijection from the set of open ideals of $\Im$ to the set of open ideals of $\Im^{*}$. (3) If $A$ is an open ideal of $\Im$, then $\Im / A \cong$ $\Im^{*} / A^{*}$ as unital quadratic Jordan algebras.

Let $K$ be an ideal of the unital quadratic Jordan algebra $\Im$ and define $K^{(0)}=\Im, K^{(1)}=K$, and, for $n \geqslant 1, K^{(n+1)}=U_{K}(n) K^{(n)}$. Then the following lemma is derived directly from the fact, proved by Tsai and Foster in [11], that $A$ and $B$ ideals of $\Im \Rightarrow U_{A} B$ is an ideal of $\Im$.

LEMMA 2. If $K$ is an ideal of $\Im$, then $K^{(n)}$ is an ideal and $K^{(n+1)} \subseteq$ $K^{(n)}$ for every nonnegative integer $n$.

Thus for any ideal $K$ of $\mathcal{Y}$ a topology is induced by the nonincreasing sequence of ideals $\left\{K^{(i)}\right\} . K$ is called a nucleus for $\}$ if $\bigcap_{i} K^{(i)}=0$, and the topology so induced is called the $K$-topology for $\Im$.

Let $\mathcal{Y}^{*}$ be the completion of $\mathcal{Y}$ with respect to a $K$-topology. Then $K^{*}=K^{(1) *}$ is a nucleus for $\Im^{*}$, but the completion topology for $\Im^{*}$ is induced by the sequence of ideals $\left\{K^{(i) *}\right\}$ which is not (a priori) the same as the $K^{*}$-topology, for the $K^{*}$-topology need not even be complete. However, $K^{*(n)} \subseteq$ $K^{(n) *}$ for every nonnegative integer $n$ since it is clear for $n=0,1$, and $z^{*} \in$ $K^{*(2)} \Rightarrow z^{*}$ is the sum of terms of the form $U_{x^{*}}^{*} y^{*}, x^{*}, y^{*} \in K^{*} \Rightarrow x^{*}=$ $\lim x_{i}, y^{*}=\lim y_{i}$ are limits of sequences on $K \Rightarrow U_{x^{*}}^{*} y^{*}=\lim U_{x_{i}} y_{i} \in K^{(2) *}$ so if $K^{*(i)} \subseteq K^{(i) *}$, then

$$
K^{*(i+1)}=\left(K^{*(i)}\right)^{(2)} \subseteq\left(K^{(i) *}\right)^{(2)} \subseteq\left(K^{(i)}\right)^{(2) *}=K^{(i+1) *}
$$

and the relation follows by induction. Hence the norm induced by the completion of $3^{*}$ is less than or equal to the norm induced by the $K^{*}$-topology. 
DEFInITION. A unital quadratic Jordan algebra $\mathcal{Y}$ is a local Jordan algebra if (1) $\operatorname{Rad} \Im$ is a maximal ideal, (2) $\bigcap_{i} \operatorname{Rad}(\Im)^{(i)}=0$, and (3) $\left.\Im / \operatorname{Rad}\right\}$ satisfies the descending chain condition for principal inner ideals (DCC).

Let $\mathcal{U}$ be a unital associative algebra and let $\Im$ be any subalgebra (with 1 ) of $\mathfrak{A}^{+}$. If $K$ is an ideal of $\mathfrak{Y}$ then, for $x, y \in K, U_{x} y=x y x \in K^{3}$. Thus by induction $K^{(i)} \subseteq K^{3^{i-1}}$ for $i \geqslant 1$. If $\mathscr{A}$ is commutative and $1 / 2 \in \Phi$, then $x, y, z$ in $K$ implies

$$
x y z=1 / 2(x y z+z y x)=U_{x, z} y=U_{x+z} y-U_{x} y-U_{z} y \in K^{(2)} .
$$

Thus in this case $K^{(i)}=K^{3^{i-1}}$ for $i \geqslant 1$.

Now $\mathfrak{A}$ is a local associative algebra, if $\mathscr{U}$ is a unital associative algebra such that $\bigcap_{i} \operatorname{Rad}(\mathscr{U})^{i}=0, \operatorname{Rad} \mathscr{U}$ is a maximal ideal, and $\mathscr{U} / \operatorname{Rad} \mathscr{U}$ is right Artinian. We note that $\operatorname{Rad}\left(\mathscr{Q}^{+}\right)=\operatorname{Rad} \mathscr{A}\left[8\right.$, p. 382] and $\bigcap_{i} \operatorname{Rad}\left(\mathscr{X}^{+}\right)^{(i)}=$ 0 by the remarks from the preceding paragraph. Furthermore $\mathfrak{U}^{+} / \operatorname{Rad}\left(\mathscr{A}^{+}\right) \cong$ $(\mathfrak{Q} / \operatorname{Rad} \mathscr{\mathscr { C }})^{+}$where the latter is simple $\left[8\right.$, p. 384] so $\operatorname{Rad}\left(\mathfrak{Q}^{+}\right)$is a maximal ideal of $\mathfrak{U}^{+}$. Finally $\mathfrak{U}^{+} / \operatorname{Rad}\left(\mathfrak{U}^{+}\right)$satisfies the DCC $[4$, p. 3.17], and therefore $\mathfrak{A}^{+}$is a local Jordan algebra. Moreover, if $1 / 2 \in \Phi, \mathfrak{Y}^{\circ}$ is an associative local Jordan algebra $\Leftrightarrow \mathcal{Z}$ is a local commutative associative algebra.

Let $\Im$ be a local Jordan algebra with $\Im^{*}$ the completion of $\Im$ with respect to the nucleus $\operatorname{Rad} \Im$, hereafter called the completion of $\Im$. We will establish that $\mathcal{S}^{*}$ is also a local Jordan algebra after showing that the closure of $\operatorname{Rad} \mathcal{Y}$ in $\mathcal{Y}^{*}$ coincides with the radical of the completion of $\mathcal{Y}^{*}$.

REMARK. Since $U_{x^{m}}\left(x^{n}\right)=x^{n+2 m}$ holds in special Jordan algebras, it holds in any Jordan algebra $\mathcal{Y}$, and for $x \in K, K$ an ideal of $\Im, x^{3^{k-1}} \in K^{(k)}, k$ any positive integer, follows by induction on $k$. Thus for $m \geqslant 3^{k}$,

$$
U_{x^{3-1}}\left(x^{m-2 \cdot 3^{k-1}}\right) \in K^{(k)} .
$$

Hence, if $K$ is a nucleus and $z \in K(\|z\|<1)$, then $\left\|z^{n}\right\| \leqslant e^{-\left[\log _{3} n\right]}$, so $\lim z^{n}=0$.

THEOREM 1. If $\Im$ is a local Jordan algebra with completion $\Im^{*}$, then $\left(\operatorname{Rad} 3^{*}\right)^{*}=\operatorname{Rad}\left(3^{*}\right)$.

Proof. Since $\operatorname{Rad} \mathcal{Y}$ is open in the $\operatorname{Rad} \mathcal{Y}$-topology, $\mathcal{3} / \operatorname{Rad} \mathcal{Z} \cong$ $\Im^{*} /\left(\operatorname{Rad} \Im^{*}\right.$ by Lemma $1(3)$. Thus $\Im^{*} /\left(\operatorname{Rad} \Im^{*}\right.$ is semisimple so $\operatorname{Rad}\left(\Im^{*}\right)$ $\subseteq(\operatorname{Rad} \Im)^{*}$. Conversely, $z \in(\operatorname{Rad} \Im)^{*}$ implies $\|z\|<1$, hence $\lim z^{i}=0$ and the standard proof shows that $1-z$ has inverse $\Sigma_{0<i<\infty} z^{i}$. Thus $\left(\operatorname{Rad} \Im^{*} \subseteq \operatorname{Rad}\left(\Im^{*}\right)\right.$.

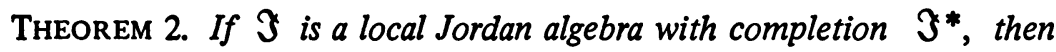
$\Im^{*}$ is a local Jordan algebra. 
Proof. Since $\Im / \operatorname{Rad} \Im \cong \Im^{*} /(\operatorname{Rad} \Im)^{*}=\Im^{*} / \operatorname{Rad}\left(\Im^{*}\right)$ by Theorem 1 and $\Im / \operatorname{Rad} \mathcal{J}^{*}$ is simple and satisfies the DCC, $\Im^{*} / \operatorname{Rad}\left(\Im^{*}\right)$ also has these properties. Thus $\operatorname{Rad}\left(\Im^{*}\right)$ is a maximal ideal, $\Im^{*} / \operatorname{Rad}\left(\Im^{*}\right)$ satisfies the DCC, and $R=\bigcap_{i} \operatorname{Rad}\left(\Im^{*}\right)^{(i)}=0$ since $\bigcap_{i} \operatorname{Rad}\left(\Im^{(i)}=0 \Rightarrow \bigcap_{i}\left(\operatorname{Rad} \Im^{(i)}\right)^{*}=0\right.$ whereas the latter contains $R$.

In the last two sections we turn to the determination of the structure of local Jordan algebras over fields of characteristic not 2, which are already complete in the $\operatorname{Rad} \Im$-topology. Warning: the completion of $\mathcal{Y}$ is complete only in the completion topology induced by the $\left(\operatorname{Rad}\left(\mathcal{J}^{(i)}\right)^{*}=\left(\operatorname{Rad}\left(\mathcal{J}^{*}\right)^{(i)}\right)^{*}\right.$, and not necessarily in the $\operatorname{Rad}\left(\Im^{*}\right)$-topology induced by the $\operatorname{Rad}\left(\Im^{*}\right)^{(i)}$. Thus the completion of a local Jordan algebra is not necessarily a complete local Jordan algebra. However, it can be verified that essentially the same structure theory prevails.

3. Idempotent lifting. We say that $\mathcal{Y}$ is a complete local Jordan algebra if $\mathcal{Y}$ is a local Jordan algebra which is complete relative to the $\mathrm{Rad} \mathcal{Y}$-topology. In the final section we will see that the structure of these algebras over a field of characteristic not 2 is heavily dependent upon the second coordinatization theorem $[3$, p. 137], which can be invoked because of the idempotent lifting property they exhibit.

Hereafter we assume that $\Phi$ is a field and observe that a bar will always mean modulo $\operatorname{Rad} 3$. Furthermore, since $\|z\|<1$ implies that any power series in $z$ converges, $\Phi \llbracket z \rrbracket$ is contained in any closed subset of $\}$ containing $\Phi[z]$.

Proposition 1. Let $K$ be a closed subalgebra of the complete local Jordan algebra 3 . If $f \in K$ and $\bar{f}$ is a nonzero idempotent of $\overline{\mathcal{Y}}$, then there is a nonzero idempotent $e \in K$ with $\bar{e}=\bar{f}$.

Proof. We let $s$ be an indeterminate and $\mathscr{U}=\Phi s+\Phi \llbracket t \rrbracket$ where $t=$ $s^{2}-s \in \Phi \llbracket s \rrbracket$. Then $w=-t(1+4 t)^{-1} \in \Phi \llbracket t \rrbracket$, and there exists $x \in \Phi \llbracket t \rrbracket$ such that $x^{2}-x=w\left(\right.$ take $x=\Sigma_{1<i<\infty} \alpha_{i}(-w)^{i}$ with $\alpha_{1}=1$ and $\alpha_{i+1}=$ $\left.\Sigma_{1<j<i} \alpha_{j} \alpha_{i-j+1}\right)$. Hence $u=s-x s-s x+x$ is an idempotent in $\Phi \llbracket s \rrbracket[5$, p. 72]. Let $\theta: \mathfrak{Q}^{+} \rightarrow \Phi f+\Phi \llbracket z \rrbracket\left(z=f^{2}-f\right)$ be determined by $s \mapsto f$ and $t \mapsto z$. Then $\theta$ is a Jordan homomorphism so $e=f-(\theta x) \circ f+\theta x=\theta u$ is an idempotent in $\Im$.

Now $z=f^{2}-f \in \operatorname{Rad} \Im$ and $f \in K \Rightarrow z \in \operatorname{Rad} \Im \cap K$. Thus each of $\operatorname{Rad} \Im$ and $K$ a closed subalgebra implies $\Phi \llbracket z \rrbracket \subseteq \operatorname{Rad} \Im \cap K$. Therefore $\theta x \in \operatorname{Rad} \mathcal{J} \cap K$ so $\bar{e}=\bar{f}$ and $e \in K$.

Proposition 2. Let $\}$ be a complete local Jordan algebra. If $e^{2}=e \in$ $3, \bar{f}^{2}=\bar{f}$, and $\bar{e} \perp \bar{f}$, then there is an idempotent $g \in \mathcal{Y}$ with $e \perp g$ and $\bar{g}=\bar{f}$. 
Proof. Let $\Im=\Im_{0} \oplus \Im_{1 / 2} \oplus \Im_{1}$ be the Peirce decomposition of $\Im_{\text {rel- }}$ ative to $e$. Without loss of generality we may assume $f \in \Im_{0} \quad\left(f^{\prime}=U_{1-e} f \in\right.$ $\Im_{0}$ and $\bar{f}^{\prime}=\bar{f}$ ). Since $U_{1-e}$ is a bounded idempotent linear operator, $\Im_{0}=$ $U_{1-e} \Im$ is a closed inner ideal of $\Im$ and an application of Proposition 1 yields an idempotent $g \in \Im_{0}$ with $\bar{g}=\bar{f}$. Clearly $e \perp g$.

We can now establish the first of two main lemmas.

Lemma 3. Let $\}$ be a complete local Jordan algebra. If $\left\{\bar{f}_{1}, \bar{f}_{2}, \cdots\right.$, $\left.\bar{f}_{n}\right\}$ is a supplementary set of pariwise orthogonal idempotents, then there exists a supplementary set $\left\{e_{1}, e_{2}, \cdots, e_{n}\right\}$ of pairwise orthogonal idempotents in $\Im$ such that $\bar{e}_{i}=\bar{f}_{i}$ for $1 \leqslant i \leqslant n$.

Proof. The standard induction suffices to show the existence of a set of pairwise orthogonal idempotents $\left\{e_{1}, e_{2}, \cdots, e_{n}\right\}$ such that for each $i, \bar{e}_{i}=$ $\bar{f}_{i}$ [5, p. 73]. Now assume $\Sigma_{i} f_{i}=e$. Then $\bar{e}=\overline{1}$ so $\bar{e}$ is invertible. Hence $e$ is an invertible idempotent and thus $e=1$.

The importance of Lemma 3 becomes apparent in Proposition 3 to which we shall turn after recalling some facts about Jordan matrix algebras found in [3]. It now becomes necessary to assume that the characteristic of $\Phi$ is not 2 and we do so throughout the remainder of the paper. If $(D, j)$ is a unital algebra with involution $j, D_{n}$ is the algebra of $n \times n$ matrices over $D$, and $a$ is any diagonal matrix in $D_{n}$ whose diagonal entries are symmetric and invertible in the nucleus of $D(=\{x \in D:[x, b, c]=[b, x, c]=[b, c, x]$ for all $b, c \in D\})$, then the symmetric elements $\mathfrak{Q}\left(D_{n}, J_{a}\right)$ of $\left(D_{n}, J_{a}\right)$ relative to the canonical involution $J_{a}: X \mapsto a^{-1} \hat{X}^{t} a$ ( $\hat{X}^{t}$ the conjugate transpose of $X$ ) with product $X \cdot Y=1 / 2(X Y+Y X)$ form a Jordan matrix algebra of order $n$, providing $\mathfrak{Q}\left(D_{n}, J_{a}\right)$ is a linear Jordan algebra. For $n \geqslant 3, \mathfrak{Q}\left(D_{n}, J_{a}\right)$ is a linear Jordan algebra if and only if $D$ is alternative with symmetric elements in the nucleus. Throughout what follows, the notation of [3] will be used; in particular, if $x e_{i j}$ is the matrix with $x$ in the $(i, j)$ position and 0 's elsewhere, then $x[i j]=$ $x e_{i j}+\left(x e_{i j}\right)^{J}$.

Suppose $\mathfrak{Q}=\mathfrak{Q}\left(D_{n}, J_{a}\right)$ is a Jordan matrix algebra with $n \geqslant 3$, and let $E$ be the ideal of $D$ such that $\operatorname{Rad} \mathfrak{Q}=E_{n} \cap \mathfrak{Q}$. Then $z \in E \Rightarrow z[12] \in$ $\operatorname{Rad} \mathfrak{Q}$ and if $u=1[12]+\Sigma_{3 \leqslant i \leqslant n} e_{i i}$, then $u$ is invertible in $\mathfrak{Q}$ so $u-$ $z$ [12] $=(1-z)[12]+\Sigma_{3<i<n} e_{i i}$ is invertible in $\mathfrak{Q}$ [7, p. 672] with inverse $(1-z)^{-1}[21]+\Sigma_{3 \leqslant i \leqslant n} e_{i i} \Rightarrow z$ is quasi-invertible $\Rightarrow E \subseteq \operatorname{Rad} D \Rightarrow \operatorname{Rad} \mathfrak{\mathbb { C }} \subseteq$ $(\operatorname{Rad} D)_{n} \cap \mathfrak{Q}$. Thus if $\operatorname{Rad} \mathfrak{W}$ is a maximal ideal, $\operatorname{Rad} \mathfrak{Q}=(\operatorname{Rad} D)_{n} \cap \mathfrak{Q}$.

Proposition 3. Let 3 be a complete local Jordan algebra such that $\bar{\jmath} \cong \$\left(\bar{D}_{n}, J_{\bar{a}}\right)$, a Jordan matrix algebra of order $n \geqslant 3$. Then $\mathcal{Y}$ is isomorphic to a Jordan matrix algebra $\mathfrak{S}\left(D_{n}, J_{a}\right)$ where the ideal in $\mathfrak{(}\left(D_{n}, J_{a}\right)$ corresponding 
to $\operatorname{Rad} \mathfrak{Y}$ is $(\operatorname{Rad} D)_{n} \cap \mathfrak{S}$ with $\bar{D}=D / \operatorname{Rad} D$.

Proof. First suppose $\mathfrak{Y} \cong \mathfrak{S}\left(D_{n}, J_{a}\right)=\mathfrak{Q}$. Then $\mathfrak{Q}$ is a local Jordan algebra so $\operatorname{Rad} \mathfrak{S}=(\operatorname{Rad} D)_{n} \cap \mathfrak{S}$.

Next assume $\sigma$ to be an isomorphism from $\bar{\zeta}$ onto $\mathfrak{Q}\left(\bar{D}_{n}, J_{\bar{a}}\right)$ and let $\bar{e}_{i}, 1 \leqslant i \leqslant n$, be elements of $\bar{\Im}$ such that $\sigma \bar{e}_{i}=1 / 2[i i]$. By Lemma 3 , we may assume the set $\left\{e_{1}, e_{2}, \cdots, e_{n}\right\}$ to consist of supplementary pairwise orthogonal idempotents of $\Im$. To use the second coordinatization theorem it suffices to know that $e_{1}$ and $e_{j}$ are connected, $1 \leqslant j \leqslant n$, and the proof here differs from that of Theorem 10 in [3, p. 151] only in the method of proving this fact.

Now the $\bar{e}_{i}$ are connected so there exist $\bar{u}_{1 j}, 1 \leqslant j \leqslant n$, in the Peirce spaces $\bar{\Im}_{1 j}$ such that $\sigma \bar{u}_{1 j}=1[1 j]$ and $\bar{u}_{1 j}$ is invertible in $U_{\bar{e}} \bar{\Im}=\bar{\Im}_{11} \oplus$ $\bar{\Im}_{1 j} \oplus \bar{\Im}_{j j}\left(e=e_{1}+e_{j}\right)$. Let $v_{1 j}=U_{e_{1}, e_{j} u_{1 j}}$ so that $v_{1 j} \in \Im_{1 j}$. Since $\bar{v}_{1 j}=\bar{u}_{1 j}$ is invertible, $v_{1 j}$ is invertible modulo $\operatorname{Rad} U_{e} \Im=\operatorname{Rad} \Im \cap U_{e} \Im$ $\left[9\right.$, p. 10] and thus $v_{1 j}$ is invertible in $U_{e} \Im$. Therefore $e_{1}$ and $e_{j}$ are connected for each $j$ and the result follows.

If $B$ is an ideal of the alternative algebra $D$, we may define certain "powers" of $B$ as follows: let $B^{[0]}=D ; B^{[1]}=B ; B^{\left[3^{m+1}\right]}=$ $\left(B^{\left[3^{m}\right]}\right)^{2} B^{\left[3^{m}\right]}+B^{\left[3^{m}\right]}\left(B^{\left[3^{m}\right]}\right)^{2}$. Since the sum and product of two ideals is an ideal, $B^{\left[3^{m}\right]}$ is an ideal for $0 \leqslant m<\infty$. Clearly, if $D$ is associative, then $B^{\left[3^{m}\right]}=B^{3^{m}}$. We now close this section by establishing an important lemma which identifies the $\operatorname{Rad}(\Im)^{(k)}$ for $\mathcal{Y}=\mathfrak{Q}\left(D_{n}, J_{a}\right)$.

Lemma 4. Let $\mathfrak{S}=\mathfrak{S}\left(D_{n}, J_{a}\right)$ be a Jordan matrix algebra of order $n \geqslant$ 3 and assume $\operatorname{Rad} D=A(1)$, where $\operatorname{Rad}(\mathfrak{W})^{(k)}=A(k)_{n} \cap \mathfrak{\text { . Then }} A(k)=$ $\operatorname{Rad}(D)^{\left[3^{k-1}\right]}$ for $1 \leqslant k<\infty$ and $\bigcap_{k} \operatorname{Rad}(\mathfrak{Q})^{(k)}=0$ if and only if $\bigcap_{k} A(k)=0$.

Proof. In view of the lattice isomorphism between ideals of $\mathfrak{Q}$ and ideals of $(D, j)[3$, p. 129], there are ideals $A(k)$ of $(D, j), 1 \leqslant k<\infty$, such that $\operatorname{Rad}(\mathfrak{Q})^{(k)}=A(k)_{n} \cap \mathfrak{W}$. Thus $\bigcap_{k} \operatorname{Rad}(\mathfrak{W})^{(k)}=\left(\bigcap_{k} A(k)\right)_{n} \cap \mathfrak{W}$, where $B_{n} \cap \mathfrak{Q}=0 \Leftrightarrow B=0, B$ an ideal of $(D, j)$. This completes the proof of the last statement.

Now assume $A(1)=\operatorname{Rad} D$. For example, we have seen this will be the case if $\operatorname{Rad} \mathfrak{S}$ is a maximal ideal, so in particular if $\mathfrak{Q}$ is local. Then we need to know that whenever $K=B_{n} \cap \mathfrak{S}, L=B_{n}^{\prime} \cap \mathfrak{Q}$, and $U_{K} L=B_{n}^{\prime \prime} \cap \mathfrak{Q}, K$ and $L$ ideals of $\mathfrak{Q}$, then $B^{\prime \prime}=\left(B B^{\prime}\right) B+B\left(B^{\prime} B\right)$. By definition of the operation $U_{X} Y=2(Y \cdot X) \cdot X-Y \cdot X^{2}$ in $\mathfrak{Q}$, all entries of linear combinations of such products are in either $\left(B B^{\prime}\right) B$ or $B\left(B^{\prime} B\right)$ so $B^{\prime \prime} \subseteq\left(B B^{\prime}\right) B+B\left(B^{\prime} B\right)$. Conversely, if $b_{1}, b_{2} \in B$ and $b^{\prime} \in B^{\prime}$, then by QM9 [4, p. 2.15],

$$
\left(b_{1} b^{\prime}\right) b_{2}[13]=U_{b_{1}[13], b_{2}[23]} b^{\prime}[32]
$$


and

$$
b_{1}\left(b^{\prime} b_{2}\right)[13]=U_{b_{1}[12], b_{2}[13]} b^{\prime}[21] \in U_{K} L
$$

so $\left(B B^{\prime}\right) B+B\left(B^{\prime} B\right) \subseteq B^{\prime \prime}$.

4. Structure theorem. Recall that in an arbitrary unital quadratic Jordan algebra $\mathcal{Y}$, a nonzero idempotent $e$ is completely primitive if $U_{e} \mathcal{Y}$ is a Jordan division algebra. $\Im$ has finite capacity if it contains a finite supplementary set of pairwise orthogonal completely primitive idempotents and the minimum number of such idempotents is called the capacity of $\Im$.

Let $\mathcal{Y}$ be a complete local Jordan algebra over a field of characteristic not 2. Then $\bar{\Im}=\Im / \operatorname{Rad} \Im$ is simple and satisfies the DCC so the structure of $\bar{\Im}$ is given by the second structure theorem $[3$, p. 179]. Since $\bar{\Im}$ contains a supplementary set $\left\{\bar{e}_{1}, \bar{e}_{2}, \cdots, \bar{e}_{n}\right\}$ of pairwise orthogonal completely primitive idempotents, by Lemma 3 we may assume $\left\{e_{1}, e_{2}, \cdots, e_{n}\right\}$ to be a supplementary set of pairwise orthogonal idempotents in $\mathcal{\Im}$. Clearly $\overline{\mathcal{Y}}$ has capacity $n$ so we classify the algebras according to these capacities. We say that $\Im$ is completely primary if $\Im / \operatorname{Rad} \Im$ has capacity 1 . By $(2.2)$ and $[3, \mathrm{p} .158]$, the following are equivalent: (1) $\mathcal{Y}$ is completely primary; (2) $\overline{\mathcal{Y}}$ is a Jordan division algebra; (3) $\operatorname{Rad} \mathcal{Y}$ is the set of noninvertibles in $\Im$.

If $n=1$, then $\mathcal{Y}$ is a complete completely primary local Jordan algebra.

If $n=2$, write $\Im=\Im_{11} \oplus \Im_{12} \oplus \Im_{22}$ as the sum of the Peirce spaces relative to $e_{1}$ and $e_{2}$. For $i=1,2, \Im_{i i} / \operatorname{Rad} \Im_{i i}$ is a Jordan division algebra and

$$
\bigcap_{k} \operatorname{Rad}\left(\Im_{i i}\right)^{(k)}=\bigcap_{k}\left(\operatorname{Rad} \Im \cap \Im_{i i}\right)^{(k)} \subseteq \bigcap_{k} \operatorname{Rad}\left(\Im^{(k)}=0\right.
$$

so each $\Im_{i i}$ is a completely primary local Jordan algebra. Furthermore, each $\Im_{i j}$ is complete in the subspace topology since it is closed.

Now assume $n \geqslant 3$. Then $\overline{\mathcal{Y}} \cong \$\left(\bar{D}_{n}, J_{\bar{a}}\right)$, a Jordan matrix algebra, and $(\bar{D}, j)$ is either simple as an associative algebra with involution or an algebra of octonions over an extension field with standard involution ( $n=3$ only). By Proposition 3, $\Im \cong \$\left(D_{n}, J_{a}\right)$ and the ideal of $(D, j)$ which corresponds to $\operatorname{Rad} \mathcal{Y}$ under the given isomorphism is $\operatorname{Rad} D$.

Assume that $D$ is associative (note $\bar{D}$ cannot be an algebra of octonions). In each of the possible cases $\bar{D}$ is Artinian and either is simple or has two ideals. Thus $D$ has at most two ideals containing $\operatorname{Rad} D$, and $D$ is unital. By Lemma $4, \bigcap_{k} \operatorname{Rad}(D)^{3^{k-1}}=0$ so $\bigcap_{k} \operatorname{Rad}(D)^{k}=0$. Hence $D$ is a semilocal associative algebra which is not necessarily Noetherian.

We now drop the assumption that $D$ be associative and let the ideal $A(k)$ of $(D, j)$ be determined as in Lemma 4. Then $\bigcap_{k} A(k)=0$ so $D$ is a metric 
space with respect to the induced topology. Now $D$ is isometrically isomorphic to $\Im_{12}$ as a vector space where $\Im_{12}$ is closed $\left(\Im_{12}=U_{e_{1}, e_{2}} \Im, U_{e_{1}, e_{2}}\right.$ a bounded idempotent linear operator). Therefore $\Im_{12}$ is complete in the subspace topology which coincides with the topology induced by $\{A(k)\}$ so that $D$ is complete in the induced topology. If $D$ is associative, then this coincides with the $\operatorname{Rad} D$-topology.

If $n \neq 2$, the converse holds. For $\Im$ a complete completely primary Jordan algebra, $\operatorname{Rad} \mathcal{Y}$ is a nucleus, $\overline{\mathcal{Y}}$ satisfies the DCC as a Jordan division algebra, and $\operatorname{Rad} \mathcal{Y}$ is a maximal ideal.

Next assume $\Im=\mathfrak{D}\left(D_{n}, J_{a}\right), n \geqslant 3$, where $D$ is a complete semilocal associative algebra such that $\operatorname{Rad} D$ is the unique maximal $j$-invariant ideal of $D$. Then $\operatorname{Rad} \mathcal{Y}=(\operatorname{Rad} D)_{n} \cap \mathcal{Y}$ is the unique maximal ideal of $\mathcal{Y}$ because of the ideal lattice isomorphism, and $\bigcap_{k} \operatorname{Rad}(D)^{k}=0$ implies $\bigcap_{k} \operatorname{Rad}(\Im)^{(k)}=$ 0 by Lemma 4. Since $\bar{D}=D / \operatorname{Rad} D$ is simple Artinian with involution and $\bar{\Im} \cong \mathfrak{P}\left(\bar{D}_{n}, J_{\bar{a}}\right)$ by Theorem 3 in $[3$, p. 130], $\Im$ satisfies the DCC by the second structure theorem. Thus $\Im$ is a local Jordan algebra.

Since $D$ is complete in the topology induced by the sequence $\left\{\operatorname{Rad}(D)^{3^{k-1}}\right\}$, $D_{n}$ is complete in the topology induced by the sequence $\left\{\left(\operatorname{Rad}(D)^{3^{k-1}}\right)_{n}\right\}$. Since $\Im=\$\left(D_{n}, J_{a}\right)$ is closed in this topology, $\Im$ is complete in the subspace topology induced by the sequence $\left\{\left(\operatorname{Rad}(D)^{3^{k-1}}\right)_{n} \cap \Im\right\}=\left\{\operatorname{Rad}(\Im)^{(k)}\right\}$ (Lemma 4). Thus $\mathcal{Y}$ is a complete local Jordan algebra.

If $n=3$ and $(D, j)$ is alternative with $\operatorname{Rad} D$ the unique maximal ideal of $(D, j)$ such that $D$ is complete in the topology induced by the sequence $\{A(k)\}$, where $A(k)=\operatorname{Rad}(D)^{\left[3^{k-1}\right]}$ and $\operatorname{Rad}(\Im)^{(k)}=A(k)_{n} \cap \Im$, then $\operatorname{Rad} \mathcal{Y}$ is a maximal ideal, $\bigcap_{k} \operatorname{Rad}(\Im)^{(k)}=0$ and $\Im$ is complete in the $\operatorname{Rad} \Im$-topology as before. If the nonzero symmetric elements of $(\bar{D}, j)$ are invertible in the nucleus of $\bar{D}$, then $\bar{\Im} \cong \$\left(\bar{D}_{n}, J_{\bar{a}}\right)$ satisfies the DCC by the second structure theorem. Therefore $\mathcal{Y}$ is a complete local Jordan algebra.

We summarize these results in the following theorem.

THEOREM 3. If $\}$ is a complete local Jordan algebra over a field of characteristic not 2 , then $\bar{\Im}$ has finite capacity $n$. Hence:

(1) If $n=1$, then $\Im$ is a complete completely primary (local) Jordan algebra.

(2) If $n=2$, then $\Im_{1} \Im_{1} \oplus \Im_{2} \oplus S$, where $\Im_{1}$ and $\Im_{2}$ are completely primary local Jordan algebras, $S$ is a subspace of $\mathcal{Y}$, and each is complete in the induced topology.

(3) If $n \geqslant 3$, then $\Im \cong \$\left(D_{n}, J_{a}\right)$, a Jordan matrix algebra, such that $\operatorname{Rad} \$\left(D_{n}, J_{a}\right)=(\operatorname{Rad} D)_{n} \cap \$\left(D_{n}, J_{a}\right)$, and either

(i) $(D, j)$ is a unital not associative alternative algebra with involution, 
$\operatorname{Rad} D$ is the unique maximal ideal of $(D, j),(D / \operatorname{Rad} D, j)$ has its nonzero symmetric elements invertible in its nucleus, and $D$ is complete in the topology induced by the sequence of ideals $\{A(k)\}$ of $(D, j)$ where $\operatorname{Rad} \mathfrak{S}\left(D_{n}, J_{a}\right)^{(k)}=$ $A(k)_{n} \cap \mathfrak{W}\left(D_{n}, J_{a}\right) \quad(n=3$ only $)$ or

(ii) $(D, j)$ is an associative algebra with involution which is a complete semilocal algebra such that $\operatorname{Rad} D$ is the unique maximal ideal of $(D, j)$.

Conversely, if $\Im$ is a complete completely primary Jordan algebra as in (1) or $\mathcal{J}=\mathfrak{Q}\left(D_{n}, J_{a}\right)$, a Jordan matrix algebra of order $n \geqslant 3$ as in (3), then $\}$ is a complete local Jordan algebra.

We remark that if $\mathcal{Y}$ is associative, then the fact that the Jordan and associative topologies coincide implies $\Im$ is a complete local Jordan algebra which is also an associative algebra if and only if $\mathcal{Y}$ is a complete local commutative associative algebra.

\section{REFERENCES}

1. E. Batho, Non-commutative semi-local and local rings, Duke Math. J. 24 (1957), 163-172. MR 19, 116.

2. I. S. Cohen, On the structure and ideal theory of complete local rings, Trans. Amer. Math. Soc. 59 (1946), 54-106. MR 7, 509.

3. N. Jacobson, Structure and representations of Jordan algebras, Amer. Math. Soc. Colloq. Publ., vol. 39, Amer. Math. Soc., Providence, R. I., 1969. MR 40 \#4330.

4. - Lectures on quadratic Jordan algebras, Tata Institute, Bombay, 1970. 34 \#5857.

5. J. Lambek, Lectures on rings and modules, Blaisdell, Waltham, Mass., 1966. MR

6. K. McCrimmon, A general theory of Jordan rings, Proc. Nat. Acad. Sci. U.S.A. 56 (1966), 1072-1079. MR 34 \#2643.

7. - The radical of a Jordan algebra, Proc. Nat. Acad. Sci. U.S.A. 62 (1969), 671-678. MR $42 \# 3137$.

8. - On Herstein's theorems relating Jordan and associative algebras, J. Algebra 13 (1969), 382-392. MR 40 \#2721.

9. - A characterization of the radical of a Jordan algebra, J. Algebra 18 (1971), 103-111. MR 43 \#3316.

10. M. Nagata, Local rings, Interscience Tracts in Pure and Appl. Math., no. 13, Interscience, New York, 1962. MR 27 \#5790.

11. C. Tsai and D. Foster, Primary ideal theory for quadratic Jordan algebras, J. Algebra 24 (1973), 439-452. MR 47 \#3477.

12. O. Zariski and P. Samuel, Commutative algebra. Vol. I, Van Nostrand, Princeton, N. J., 1958. MR 19, 833.

DEPARTMENT OF MATHEMATICS, MERCYHURST COLLEGE, ERIE, PENNSYLVANIA 16501 